\title{
Ocorrência de parasitas e comensais intestinais em crianças de escola localizada em assentamento de sem-terras em Campo Florido, Minas Gerais, Brasil
}

\author{
Occurrence of intestinal parasites and commensal organisms among \\ schoolchildren living in a 'landless farm workers' settlement \\ in Campo Florido, Minas Gerais, State Brazil
}

\author{
Patricia Ferreira1, Marcelo Ribeiro Lima', Francielle Batista Oliveira1', Maria Letícia Moreira Pereira1, \\ Leila Bitar Moukachar Ramos ${ }^{3}$, Maria das Graças Marçal ${ }^{2}$ \\ e Julia Maria Costa-Cruz ${ }^{2}$
}

\begin{abstract}
Resumo O objetivo deste estudo foi verificar a ocorrência de parasitas e comensais intestinais em crianças de escola localizada em assentamento de sem-terras em Campo Florido, Minas Gerais, Brasil. Exames coproparasitológicos realizados em 72 indivíduos evidenciaram 59,7\% de positividade, sendo identificados 4 tipos de protozoários e 5 de helmintos. Concluiu-se que há necessidade de acompanhamento das condições de saúde desta população.
\end{abstract}

Palavras-chaves: Parasitas intestinais. Epidemiologia. Zona rural. Escolares. Brasil.

Abstract The aim of this study was to verify the occurrence of intestinal parasites and commensal organisms among children attending a school located in a settlement of 'landless farm workers' in Campo Florido, Minas Gerais, Brazil. Coproparasitological analyses performed on 72 school children revealed $59.7 \%$ positivity and 4 types of protozoa and 5 types of helminths were identified. It can be concluded that it is necessary to monitor the health conditions of this population.

Key-words: Intestinal parasites. Epidemiology. Rural zone. School children. Brazil.

No município de Campo Florido, MG, situa-se um assentamento filiado ao Movimento dos Sem Terra (MST). Esta área encontra-se subdividida em 114 lotes, onde residem aproximadamente 500 indivíduos. Neste local, $80,8 \%$ das residências são de alvenaria, 11,5\% de lona $5,5 \%$ de pau a pique e $2,2 \%$ de madeira. Apenas $12 \%$ das residências de tijolos apresentavam fossas assépticas, sendo que $50 \%$ da população utiliza fossa séptica e $38 \%$ depositam seus dejetos a céu aberto. Quanto à utilização da água $65,2 \%$ obtinham-na de poços ou cisternas, $24,5 \%$ utilizam água encanada não tratada, $5,6 \%$ de rio e $4,7 \%$ de mina.

Em 72 crianças escolares, com idade de 5 a 14 anos foram feitos exames de fezes colhidos em formol a $10 \%$ e examinados pelo método de sedimentação ${ }^{5}$, em novembro de 2000 . Os exames foram realizados no Laboratório de Parasitologia do Instituto de Ciências
Biomédicas da Universidade Federal de Uberlândia e os resultados encaminhados à equipe médica que atende no local, para tratamento específico dos indivíduos parasitados.

A Tabela 1 mostra a distribuição de parasitas e comensais intestinais que ocorreram em $43(59,7 \%)$ da população estudada. $O$ parasita mais freqüente foi Giardia lamblia, identificado em 22 (30,5\%) das amostras fecais. O único caso de positividade para Schistosoma mansoni correspondia a uma criança de 7 anos de idade, do sexo masculino e foi notificado ao Serviço de Vigilância Epidemiológica. Por motivos operacionais e a exemplo de Levantamento Multicêntrico desenvolvido por Campos et al' não se utilizou metodologia específica para pesquisa de Taenia sp, Strongyloides stercoralis e Enterobius vermicularis sendo que os baixos índices encontrados para estes helmintos pode ter ocorrido em função da metodologia empregada.

\footnotetext{
1. Faculdade de Medicina da Universidade Federal de Uberlândia, Uberlândia, MG. 2. Laboratório de Parasitologia do Instituto de Ciências Biomédicas da Universidade Federal de Uberlândia, MG, Brasil. 3. Departamento de Clínica Médica da Faculdade de Medicina da Universidade Federal de Uberlândia, MG, Brasil.

Endereço para correspondência: Profa Julia Maria Costa-Cruz. Laboratório de Parasitologia do Instituto de Ciências Biomédicas/UFU. Av. Pará 1720, Umuarama, 38400-902 Uberlândia, MG, Brasil.

Telefax: 5534 3218-2333

e-mail:costacruz@ufu.br

Recebido para publicação em 5/3/2002.
} 
A Tabela 2 apresenta a distribuição da positividade de parasitas e comensais intestinais das crianças segundo série e sexo.

A ocorrência de quase $60 \%$ de infecção por parasitas e comensais intestinais aqui registrada é superior aos resultados obtidos em escolares de área rural de Rolândia, Paraná $(48,1 \%)^{4}$, Bambuí, Minas Gerais $(21,9 \%)^{7}$ e Distrito de Martinésia, Uberlândia, Minas Gerais $(17,5 \%)^{3}$. Contudo, os resultados de Campo Florido foram semelhantes aos de Kobayashi et al. ${ }^{6}$ que verificaram prevalência de $68,9 \%$ de infecção parasitária em indivíduos menores de um ano até maiores de 60 anos de idade, habitantes da zona rural do município de Holambra, São Paulo.

O aumento da densidade populacional nas áreas rurais contribui para o aumento da prevalência de infecções parasitárias ${ }^{2}$. Apesar de G. lamblia ter sido o parasita de maior freqüência, este resultado poderia ser maior uma vez que o ideal seria a execução de três exames de fezes em dias alternados. No levantamento

\begin{tabular}{|c|c|c|}
\hline \multirow[b]{2}{*}{ Parasitas ou comensais intestinais } & \multicolumn{2}{|c|}{ Crianças } \\
\hline & $\mathrm{n}^{\circ}$ & $\%$ \\
\hline Giardia lamblia & 22 & 30,5 \\
\hline Entamoeba coli & 13 & 18,0 \\
\hline Hymenolepis nana & 7 & 9,7 \\
\hline Hymenolepis diminuta & 6 & 8,3 \\
\hline Ancilostomideos & 5 & 6,9 \\
\hline Enterobius vermicularis & 5 & 6,9 \\
\hline Endolimax nana & 3 & 4,2 \\
\hline lodamoeba butschlii & 2 & 2,8 \\
\hline Schistosoma mansoni & 1 & 1,4 \\
\hline
\end{tabular}

\begin{tabular}{|c|c|c|c|c|c|c|c|c|c|}
\hline \multirow[b]{2}{*}{ População escolar } & \multicolumn{3}{|c|}{ Masculino } & \multicolumn{3}{|c|}{ Feminino } & \multicolumn{3}{|c|}{ Total } \\
\hline & $\mathrm{n}^{\circ}$ & pos & $\%$ & $\mathrm{n}^{\circ}$ & pos & $\%$ & $\mathrm{n}^{\circ}$ & pos & $\%$ \\
\hline pré-escolar & 11 & 7 & 63,6 & 10 & 6 & 60,0 & 21 & 13 & 61,9 \\
\hline $1^{\text {a }}$ série & 5 & 4 & 80,0 & 8 & 6 & 75,0 & 13 & 10 & 76,9 \\
\hline $2^{\mathrm{a}}$ série & 4 & 3 & 75,0 & 8 & 4 & 50,0 & 12 & 7 & 58,3 \\
\hline $3^{\text {a }}$ série & 10 & 5 & 50,0 & 6 & 3 & 50,0 & 16 & 8 & 50,0 \\
\hline$\underline{4^{\mathrm{a}} \text { série }}$ & 5 & 2 & 40,0 & 5 & 3 & 60,0 & 10 & 5 & 50,0 \\
\hline Total & 35 & 21 & 60,0 & 37 & 22 & 59,5 & 72 & 43 & 59,7 \\
\hline
\end{tabular}

$\mathrm{n}^{\circ}=$ número analisado. pos $=$ positivos, $\%=$ porcentagem de positividade

multicêntrico de parasitoses intestinais no Brasil ${ }^{1}$, em escolares de 7 a 14 anos, também analisando uma única amostra de fezes detectou-se no Estado de Minas Gerais $44,2 \%$ de positividade, sendo os parasitas mais freqüentes o Ascaris lumbricoides (59,5\%), Trichuris trichiura (36,6\%), G. lamblia $(23,8 \%)$ e S. mansoni $(11,6 \%)$. A ausência de infecção por $A$. lumbricoides e $T$. trichiura aqui verificada também difere dos resultados nacionais em áreas rurais como, por exemplo, taxas de infecção em torno de $40 \%$ para esses dois helmintos demonstrada por Saldiva et $a^{\beta}$.
Há mais de 25 anos Vinha ${ }^{9}$ salientou a necessidade de desenvolvimento de uma política sanitária nacional para o combate às parasitoses intestinais, uma vez que as infecções estão vinculadas ao subdesenvolvimento, a falta de saneamento ambiental, a falhas na educação e informação sanitária. Conclui-se que diante dos resultados aqui apresentados, há necessidade de acompanhamento das condições de saúde das populações localizadas em assentamentos de semterras.

\section{REFERÊNCIAS BIBLIOGRÁFICAS}

1. Campos R, Briques W, Belda Neto M, Souza JM, Katz N, Salata E, Dacal ARG, Dourado H, Castanho REP, Gurvitz R, Zingano A, Pereira GJM, Ferriolli Filho E, Camilo-Coura L, Faria JAS,
Cimermam B, Siqueira Filho JB, Prata A. Levantamento multicêntrico de parasitoses intestinais no Brasil. Rhodia - Grupo Rhône-Poulen, 1988. 
2. Chan M-S. The global burden of intestinal nematode infections fifty years on. Parasitology Today 13:438-443, 1997.

3. Ferreira CB, Marçal Jr O. Enteroparasitoses em escolares do distrito de Martinésia, Uberlândia, MG: um estudo-piloto. Revista da Sociedade Brasileira de Medicina Tropical 30:373-377, 1997.

4. Giraldi N, Vidotto O, Navarro IT, Garcia JL. Enteroparasitoses prevalence among daycare and elementary school children of municipal schools, Rolândia, PR, Brazil. Revista da Sociedade Brasileira de Medicina Tropical 34:385-387, 2001.

5. Hoffman WA, Pons JA, Janer SL. The sedimentation concentration method in Schistosomiasis mansoni. Puerto Rico Journal of Public Health 9:283-291, 1934.

6. Kobayashi J, Hasegawa H, Forli AA, Nishimura NF, Yamanaka A, Shimbukuro T, Sato Y. Prevalence of intestinal parasitic infection in five farms in Holambra, São Paulo, Brazil. Revista do Instituto de Medicina Tropical de São Paulo 37:13-18, 1995.

7. Rocha SR, Silva JG, Peixoto SV, Caldeira RL, Firmo JOA, Carvalho OS, Katz N. Avaliação da esquistossomose e de outras parasitoses intestinais, em escolares do município de Bambuí, Minas Gerais, Brasil. Revista da Sociedade Brasileira de Medicina Tropical 33:431-436, 2000.

8. Saldiva SR, Silveira AS, Philippi ST, Torres DM, Mangini AC, Dias RMD, da Silva RM, Buratini MN, Massad E. Ascaris-Trichuris association and malnutrition in Brazilian children. Paediatric and Perinatal Epidemiology 13:89-98, 1999.

9. Vinha C. Incidência no Brasil de helmintos transmitidos pelo solo. Rotina coproscópica do Ex - Departamento Nacional de Endemias Rurais. Revista Brasileira de Malariologia e Doenças Tropicais 23:3-17, 1971. 